\title{
A functional approach to quantum friction: effective action and dissipative force
}

\author{
M. Belén Farías ${ }^{1}$ a , César D. Fosco $^{2}$, Fernando C. Lombardo ${ }^{1}$, Francisco D. Mazzitelli $^{2}$, and Adrián E. Rubio López ${ }^{1}$ \\ 1 Departamento de Física Juan José Giambiagi, \\ FCEYN UBA and IFIBA CONICET-UBA, Facultad de Ciencias Exactas y Naturales, \\ Ciudad Universitaria, Pabellón I, 1428 Buenos Aires, Argentina and \\ 2 Centro Atómico Bariloche and Instituto Balseiro, \\ Comisión Nacional de Energía Atómica, 8400 Bariloche, Argentina
}

(Dated: today)

We study the Casimir friction due to the relative, uniform, lateral motion of two parallel semitransparent mirrors coupled to a vacuum real scalar field, $\phi$. We follow a functional approach, whereby nonlocal terms in the action for $\phi$, concentrated on the mirrors' locii, appear after functional integration of the microscopic degrees of freedom. This action for $\phi$, which incorporates the relevant properties of the mirrors, is then used as the starting point for two complementary evaluations: Firstly, we calculate the in-out effective action for the system, which develops an imaginary part, hence a non-vanishing probability for the decay (because of friction) of the initial vacuum state. Secondly, we evaluate another observable: the vacuum expectation value of the frictional force, using the in-in or Closed Time Path formalism. Explicit results are presented for zero-width mirrors and half-spaces, in a model where the microscopic degrees of freedom at the mirrors are a set of identical quantum harmonic oscillators, linearly coupled to $\phi$.

PACS numbers: 03.70.+k, 11.10.-z, 42.50.-p, 42.50.Pq

\footnotetext{
a mbelfarias@df.uba.ar
} 


\section{INTRODUCTION}

The quantum nature of microscopic systems may, under some special circumstances, manifest itself in the form of interesting macroscopic effects. On the other hand, one of the most distinctive features of quantum phenomena are the vacuum fluctuations, among which one of the most celebrated examples are the zero-point electromagnetic field fluctuations. These, however, do not produce any observable effect in free space. This may change drastically when non-trivial boundary conditions are imposed on the electromagnetic field: in the Casimir effect (and related phenomena), a force appears even between two neutral macroscopic bodies. Indeed, this effect can be understood as due to the fact that vacuum fluctuations induce (vacuum) currents in each object, the interaction between which results in a macroscopic force [1].

The very same quantum fluctuations may also produce qualitatively different observable effects in different set-ups. One that has received much attention is the existence of a frictional force when plane mirrors which are not in contact undergo constant-speed relative parallel motion. The phenomenon manifests itself, for instance, when the mirrors' media are lossy, since a frictional force is then generated by the phase lag between the fluctuation-induced currents, and that phase lag is not present for perfect mirrors [2]. A different situation, which also leads to friction, is due to the quantum Cerenkov effect between non-dispersive media [3] at a relative speed which surpasses a threshold determined by the speed of light in the media. In any case, the effect can be understood in terms of an exchange of virtual photons between two bodies, which in turn excite their internal degrees of freedom. This effect has been analyzed [2] (and debated [4]) at length, mainly for the case of media which fill half-spaces, shifting with constant velocity. Quantum friction has been computed using different theoretical approaches [5]. Ref.[6] contains a detailed account of the works on the subject, pointing out some contradictory results in earlier literature. Note that quantum dissipative effects on moving bodies may also be due to the excitation of real photons out of the quantum vacuum, an effect known as dynamical Casimir effect (see, for instance, Ref. [7]). The latter, however, unlike the quantum friction phenomenon, requires the existence on non-vanishing accelerations.

In this paper, we present a detailed study of quantum friction between two mirrors which undergo constant parallel speed relative motion, using functional methods. We follow two complementary approaches that, we believe, shed new light on this interesting effect from the perspective of quantum field theory. We present our study for a specific simple model, consisting of a vacuum scalar field linearly coupled to a set of uncoupled quantum harmonic oscillators which are the microscopic 'matter' degrees of freedom on the mirrors.

Our first approach here is analogous to the one presented in a previous paper by some of us [8], where dissipative effects (for either normal or parallel motions) have been analysed using an Euclidean functional integral formalism for the calculation of the effective action, the result of which is rotated back to real time. Mirrors have been represented by nonlocal coupling terms in the vacuum field action, which proceed from the integration of the microscopic degrees of freedom. It has been shown there that, indeed, an imaginary part for the in-out effective action emerged as a consequence of non-contact friction. We extend here that study in more than one direction: we present a more detailed description of the model for the microscopic degrees of freedom (namely, before integrating them out) and we analyze in detail the relation between the analytic structure of the nonlocal coupling terms in Fourier space and the presence of frictional forces, performing all the calculations in real time, and discussing the subtleties of Wick rotation. The second approach consists in the explicit computation of the frictional force from the vacuum expectation value of the energy momentum tensor. We will see that, although the problem is stationary, as the in and out vacuum states of the system do not coincide, it is necessary to use the in-in or Closed Time Path (CTP) formalism. Both the in-out and in-in functional approaches have been previously applied to the case of accelerated mirrors in Ref.[9].

Regarding the mirrors, we consider two different geometries: two infinitesimally thin mirrors (that is, twodimensional mirrors of zero width) separated by a distance $a$, and two half-spaces separated by a distance $a$.

The structure of this paper is as follows: in Section II we define the class of system that we consider in this paper and establish some of the approximations to be used. We also present a microscopic model which provides concrete realizations of the system defined above. Then, in Section III, we calculate the in-out effective action, studying the relation between its analytic structure and the existence of friction. In Section IV we calculate the frictional force, by means of the in-in vacuum expectation value of the stress tensor, using the CTP formalism. Section V contains our conclusions.

\section{THE SYSTEM}

Let us begin by defining the (real-time) action $\mathcal{S}$ for the system; it depends on the vacuum field $\phi$ and on the matter fields, denoted collectively by $\psi$, confined to the mirrors. Hence, the action naturally decomposes into three terms, 
as follows:

$$
\mathcal{S}[\phi, \psi]=\mathcal{S}_{\mathrm{v}}^{(0)}[\phi]+\mathcal{S}_{\mathrm{m}}^{(0)}[\psi]+\mathcal{S}_{\mathrm{vm}}^{(\mathrm{int})}[\phi, \psi],
$$

where $\mathcal{S}_{\mathrm{v}}^{(0)}$ is the free (i.e., no mirrors) action for the vacuum field:

$$
\mathcal{S}_{\mathrm{v}}^{(0)}[\phi]=\frac{1}{2} \int d^{4} x\left[\partial^{\mu} \phi \partial_{\mu} \phi-\left(m^{2}-i \epsilon\right) \phi^{2}\right],
$$

whilst $\mathcal{S}_{\mathrm{m}}^{(0)}$ and $\mathcal{S}_{\mathrm{vm}}^{(\mathrm{int})}$ denote the actions for the free matter field and for the $\phi-\psi$ interaction, respectively. Assuming locality of the microscopic vacuum-field/matter interaction, $\mathcal{S}_{\mathrm{vm}}^{(\mathrm{int})}$ will only depend on the vacuum field at spatial points on the regions occupied by the two mirrors, which we will denote by $L$ and $R$ (each letters will be used to denote both a mirror and the spatial region it occupies). Each mirror is assumed to have homogeneous and isotropic properties on each $x^{3}=$ constant plane, whenever $x^{3}$ is inside the mirror width. Besides, those properties are independent of $x^{3}$ inside each mirror. We adopt the convention $\hbar=c=1$.

The in-out effective action $\Gamma$ for the full system described by $\mathcal{S}$ may therefore be written in terms of the vacuum persistence amplitude, $\mathcal{Z}$, which in turn can be represented as a functional integral:

$$
e^{i \Gamma}=\mathcal{Z}=\left\langle 0_{\text {out }} \mid 0_{\text {in }}\right\rangle=\int \mathcal{D} \phi \mathcal{D} \psi e^{i \mathcal{S}[\phi, \psi]} .
$$

Rather than performing the functional integrals over matter and vacuum fields simultaneously, it is convenient to introduce the partial result of integrating out just the matter degrees of freedom at the plates:

$$
\mathcal{Z}=\int \mathcal{D} \phi e^{i \mathcal{S}_{\mathrm{v}}^{(\text {eff })}[\phi]},
$$

with $\mathcal{S}_{\mathrm{v}}^{(\mathrm{eff})}[\phi] \equiv \mathcal{S}_{\mathrm{v}}^{(0)}[\phi]+\mathcal{S}_{\mathrm{v}}^{(\mathrm{int})}[\phi]$, where the second term incorporates the effect of the matter degrees of freedom, and is given by

$$
e^{i \mathcal{S}_{\mathrm{v}}^{(\mathrm{int})}[\phi]}=\int \mathcal{D} \psi e^{i\left(\mathcal{S}_{\mathrm{m}}^{(0)}[\psi]+\mathcal{S}_{\mathrm{vm}}^{(\mathrm{int})}[\phi, \psi]\right)} .
$$

Regardless of the model used for the mirrors, based on the assumptions about the system, the general form of $\mathcal{S}_{\mathrm{v}}^{\text {(int) }}[\phi]$ will be:

$$
\mathcal{S}_{\mathrm{v}}^{(\mathrm{int})}[\phi]=\mathcal{S}_{\mathrm{v}}^{(L)}[\phi]+\mathcal{S}_{\mathrm{v}}^{(R)}[\phi]
$$

where $\mathcal{S}_{\mathrm{v}}^{(R)}[\phi]$ and $\mathcal{S}_{\mathrm{v}}^{(L)}[\phi]$ are, in general, nonlocal and non-quadratic functionals of $\phi\left(x_{\|}, x^{3}\right)$, where $x_{\|} \equiv\left(x^{0}, x^{1}, x^{2}\right)$. Because of the assumed locality of the microscopic interaction, we also know that $\mathcal{S}_{\mathrm{v}}^{(L, R)}$ will depend on $\phi\left(x_{\|}, x^{3}\right)$ only for $x^{3}$ inside the region defining the respective mirror. It is convenient to introduce, in this respect, two functions $\chi_{L}\left(x^{3}\right)$ and $\chi_{R}\left(x^{3}\right)$, respectively, which determine the regions occupied by them. For finite or infinite width mirrors: $\chi_{L, R}\left(x^{3}\right)=1$ if $x_{3}$ belongs to $L, R$, and $\chi_{L, R}\left(x^{3}\right)=0$ otherwise. For zero-width ones, they are just $\delta$ functions of the corresponding value of $x^{3}$.

Thus, under the assumption that, either exactly (as in the model we shall consider) or approximately, $\mathcal{S}_{\mathrm{v}}^{(L, R)}$ is quadratic in its respective argument, we have:

$$
\mathcal{S}_{\mathrm{v}}^{(\mathrm{int})}[\phi]=-\frac{1}{2} \int_{x, y} \phi(x) V(x, y) \phi(y)
$$

(where we introduced a shorthand notation for the two spacetime integrals) with:

$$
V(x, y)=V_{L}(x, y)+V_{R}(x, y) .
$$

and

$$
V_{L, R}(x, y)=\chi_{L, R}\left(x^{3}\right) \delta\left(x^{3}-y^{3}\right) \lambda_{L, R}\left(x_{\|}-y_{\|}\right) .
$$

The 'potentials' $V_{L, R}$ can be determined by using a concrete model, or even introduced by hand, under some specific assumptions. Nevertheless, regardless of the origin of those potentials, the $\phi$ integral becomes a Gaussian,

$$
\mathcal{Z}=\int \mathcal{D} \phi e^{-\frac{1}{2} \int_{x, y} \phi(x) A(x, y) \phi(y)},
$$


where we introduced $A(x, y)$, which may be regarded as the kernel of an (integral) operator $A$. In a Dirac bracket-like notation: $A(x, y)=\langle x|A| y\rangle$, with:

$$
A(x, y)=\left[i\left(\square_{x}+m^{2}\right)+\epsilon\right] \delta(x-y)+i V(x, y)
$$

Thus, the formal result of the integral over $\phi$ yields for $\Gamma$ :

$$
\Gamma=\frac{i}{2} \operatorname{Tr} \log A
$$

An expansion of $\Gamma$ in powers of the potentials can be performed by noting that $A=A_{0}+A_{1}$, where

$$
A_{0}(x, y)=\left[i\left(\square_{x}+m^{2}\right)+\epsilon\right] \delta(x-y)
$$

is the inverse of the free Feynman propagator $G_{F}(x-y)=-i\langle 0| T[\phi(x) \phi(y)|0\rangle$, and

$$
A_{1}(x, y)=i V(x, y) \text {. }
$$

The first contribution in this expansion which already encodes a nontrivial interaction between the two mirrors is of the second order, and has the form:

$$
\Gamma_{I}^{(2)}=-\frac{i}{2} \operatorname{Tr}\left(G_{F} V_{L} G_{F} V_{R}\right)
$$

The trace may be evaluated in momentum space, so that

$$
\Gamma_{I}^{(2)}=-\frac{i}{2} \int \frac{d^{4} p}{(2 \pi)^{4}} \frac{d^{4} q}{(2 \pi)^{4}} \tilde{G}_{F}(p) \tilde{G}_{F}(q) \tilde{V}_{L}(p, q) \tilde{V}_{R}(q, p)
$$

where $\tilde{G}(p) \equiv \frac{i}{p^{2}-m^{2}+i \epsilon}$, while the two momentum space kernels $\tilde{V}_{R, L}$ are determined by the geometry and composition of the mirrors, as well as by the relative motion between them. The advantage of using a microscopic model is that the analytic properties of the kernels will be completely determined after the integration of the matter degrees of freedom. Let us consider now, in the next subsection, how the effect of the relative motion is reflected in the potentials.

\section{A. Potentials}

Since only the relative motion of the mirrors may affect the physical results, we shall use as the reference system a laboratory frame $(L)$, where $L$ is at rest, while $R$ moves rigidly with a constant speed $u$ along any direction parallel to its homogeneity and isotropy planes, $x^{1}$ say.

Using $x^{\prime \mu}, \mu=0,1,2,3$ for coordinates fixed to the moving mirror, and assuming $|u|<<1$, we have the Galilean transformations: $x^{0}=x^{\prime 0}, x^{\prime 0}=x^{0}, x^{\prime 1}=x^{1}-u x^{0}, x^{\prime 2}=x^{2}$ and $x^{\prime 3}=x^{3}$.

For the $L$ mirror, under the assumptions we presented above, the potential necessarily has the form:

$$
V_{L}(x, y)=\chi_{L}\left(x^{3}\right) \lambda_{L}\left(x_{\|}-y_{\|}\right) \delta\left(x^{3}-y^{3}\right)
$$

where $\lambda_{L}$ may be conveniently determined by its Fourier space transformed $\tilde{\lambda}_{L}\left(k^{0}, k^{1}, k^{2}\right)$. Regarding the $R$ mirror, we note that, in a comoving reference system,

$$
V_{R}^{\prime}\left(x^{\prime}, y^{\prime}\right)=\chi_{R}\left(x^{\prime 3}\right) \lambda_{R}\left(x_{\|}^{\prime}-y_{\|}^{\prime}\right) \delta\left(x^{\prime 3}-y^{\prime 3}\right)
$$

where $\lambda_{R}$ is determined by the microscopic model at rest, since it is a comoving system object. It may be written in terms of its Fourier transform, as follows:

$$
\lambda_{R}\left(x_{\|}^{\prime}-y_{\|}^{\prime}\right)=\int \frac{d^{3} k_{\|}}{(2 \pi)^{3}} e^{i k_{\|} \cdot\left(x_{\|}^{\prime}-y_{\|}^{\prime}\right)} \tilde{\lambda}_{R}\left(k_{\|}\right)
$$

(no need to introduce primed variables for the momenta, since they are integrated, dummy variables). Note that, if the two media were identical, $\tilde{\lambda}_{R}$ above would be identical to $\tilde{\lambda}_{L}$.

The interaction between the mirrors and the vacuum field will be:

$$
\mathcal{S}_{\mathrm{v}}^{(\mathrm{int})}=-\frac{1}{2} \int_{x, y} \phi(x) V_{L}(x, y) \phi(y)-\frac{1}{2} \int_{x^{\prime}, y^{\prime}} \phi^{\prime}\left(x^{\prime}\right) V_{R}^{\prime}\left(x^{\prime}, y^{\prime}\right) \phi^{\prime}\left(y^{\prime}\right) .
$$


We have to put both potentials in the same reference system. The scalar field satisfies $\phi^{\prime}\left(x^{\prime}\right)=\phi(x)$, and $\chi_{R}$ is invariant. On the other hand:

$$
\begin{aligned}
\lambda_{R}\left(x_{\|}^{\prime}-y_{\|}^{\prime}\right) & =\int \frac{d^{3} k_{\|}}{(2 \pi)^{3}} e^{i\left[k^{0}\left(x^{\prime 0}-y^{\prime 0}\right)-k^{1}\left(x^{\prime 1}-y^{\prime 1}\right)-k^{2}\left(x^{\prime 2}-y^{\prime 2}\right)\right.} \tilde{\lambda}_{R}\left(k^{0}, k^{1}, k^{2}\right) \\
& =\int \frac{d^{3} k_{\|}}{(2 \pi)^{3}} e^{i\left[k^{0}\left(x^{0}-y^{0}\right)-k^{1}\left(x^{1}-y^{1}-u\left(x^{0}-y^{0}\right)\right)-k^{2}\left(x^{2}-y^{2}\right)\right]} \tilde{\lambda}_{R}\left(k^{0}, k^{1}, k^{2}\right) \\
& =\int \frac{d^{3} k_{\|}}{(2 \pi)^{3}} e^{i\left[k^{0}\left(x^{0}-y^{0}\right)-k^{1}\left(x^{1}-y^{1}\right)-k^{2}\left(x^{2}-y^{2}\right)\right]} \tilde{\lambda}_{R}\left(k^{0}-u k^{1}, k^{1}, k^{2}\right)
\end{aligned}
$$

where we have used the Galilean transformation and a shift of integration variables. The last line in the equation above tells us that, in the $L$ system, the $R$ mirror is described by the shifted $\tilde{\lambda}_{R}$ function:

$$
\tilde{\lambda}_{R}\left(k^{0}, k^{1}, k^{2}\right) \rightarrow \tilde{\lambda}_{R}\left(k^{0}-u k^{1}, k^{1}, k^{2}\right) .
$$

\section{B. Microscopic model for the media}

We introduce here a simple microscopic model, a concrete realization of the interaction between vacuum and matter fields, which provides a physically acceptable function $\tilde{\lambda}$. Microscopic matter degrees of freedom on the media behave as one-dimensional harmonic oscillators, one at each point of the mirror. They have generalized coordinates $Q\left(x^{0}, x^{1}, x^{2}\right)=Q\left(x_{\|}\right)$, taking values in an internal space. No coupling between the oscillators is included, and there is a linear coupling between each oscillator and the vacuum field. The interaction only occurs locally, at the spatial positions occupied by the media.

To find $\mathcal{S}_{\mathrm{v}}^{(L)}$, we consider the terms in the action depending on $Q_{L}$ (for $R$ an analogous argument will apply):

$$
\mathcal{S}_{\mathrm{m}}^{(0)}=\frac{1}{2} \int d^{4} x \chi_{L}\left(x^{3}\right)\left[\dot{Q}_{L}^{2}\left(x_{\|}\right)-\left(\Omega_{L}^{2}-i \epsilon\right) Q_{L}^{2}\left(x_{\|}\right)\right]
$$

and

$$
\mathcal{S}_{\mathrm{vm}}^{(\mathrm{int})}=g_{L} \int d^{4} x \chi_{L}\left(x^{3}\right) Q_{L}\left(x_{\|}\right) \phi(x)
$$

The integral to find $\mathcal{S}_{\mathrm{v}}^{(L)}$ is a Gaussian, and it results in the potential:

$$
V_{L}(x, y)=\chi_{L}\left(x^{3}\right) \delta\left(x^{3}-y^{3}\right) \lambda_{L}\left(x_{\|}-y_{\|}\right)
$$

with the Fourier transform of $\lambda_{L}$ given by:

$$
\tilde{\lambda}_{L}\left(k_{\|}\right)=\tilde{\lambda}_{L}\left(k^{0}\right)
$$

with

$$
\tilde{\lambda}_{L}\left(k^{0}\right)=\frac{g_{L}^{2}}{\left(k^{0}\right)^{2}-\Omega_{L}^{2}+i \epsilon} .
$$

Note that, even for this simple model, $\tilde{\lambda}_{L}$ is not analytic, since it has two poles, located at $k_{L}^{0}= \pm \sqrt{\Omega_{L}^{2}-i \epsilon} \approx$ $\pm \Omega_{L} \mp \frac{i \epsilon}{2 \Omega_{L}}$.

An important remark is in order: the mass dimensions of the coupling constant $g_{L}$ and of $Q$ are different when $\chi_{L}$ is a $\delta$ function rather than a step-like function. Indeed, in the former, $[Q]=1 / 2$ and $\left[g_{L}\right]=-3 / 2$, while in the latter $[Q]=1$ and $\left[g_{L}\right]=-2$. For a moving $R$ mirror, on the other hand, we shall have

$$
\tilde{\lambda}_{R}\left(k^{0}, k^{1}\right)=\frac{g_{R}^{2}}{\left(k^{0}-u k^{1}\right)^{2}-\Omega_{R}^{2}+i \epsilon} .
$$




\section{IN-OUT EFFECTIVE ACTION}

Let us now compute the effective action as a function of the $\tilde{\lambda}$ functions which characterize the material:

$$
\Gamma_{I}^{(2)}=\frac{-i T \Sigma}{2(2 \pi)^{2}} \int d^{3} p_{\|} \tilde{\lambda}_{L}\left(p^{0}\right) \tilde{\lambda}_{R}\left(p^{0}-u p^{1}\right) \int d x^{3} d y^{3} \chi_{L}\left(x^{3}\right)\left[G\left(p_{\|}, x^{3}-y^{3}\right)\right]^{2} \chi_{R}\left(y^{3}\right),
$$

where $T$ is the total time, $\Sigma$ the total surface of the plates, and:

$$
G\left(p_{\|}, x^{3}\right)=i \int d p^{3} \frac{e^{i p^{3} x^{3}}}{\left(p_{\|}\right)^{2}-\left(p^{3}\right)^{2}+i \epsilon} .
$$

For two zero-width mirrors, at a distance $a$, we obtain:

$$
\Gamma_{I}^{(2)}=\frac{i T \Sigma}{4} \int d^{3} p_{\|} \frac{e^{2 i a \sqrt{\left(p_{\|}\right)^{2}+i \epsilon}}}{\left(p_{\|}\right)^{2}+i \epsilon} \tilde{\lambda}_{L}\left(p^{0}\right) \tilde{\lambda}_{R}\left(p^{0}-u p^{1}\right) .
$$

Here, each $\tilde{\lambda}(\omega)$ appears evaluated at a frequency measured at the rest frame of each plate. that

On the other hand, for infinite media filling half-spaces, namely, $\chi_{L}\left(x^{3}\right)=\theta\left(-x^{3}\right)$ and $\chi_{R}\left(x^{3}\right)=\theta\left(x^{3}-a\right)$, we see

$$
\Gamma_{I}^{(2)}=\frac{i T \Sigma}{4} \int d^{3} p_{\|} \frac{\tilde{\lambda}_{L}\left(p^{0}\right) \tilde{\lambda}_{R}\left(p^{0}-u p^{1}\right)}{\left(p_{\|}\right)^{2}+i \epsilon} \int d x_{3} d x_{3}^{\prime} \theta\left(-x_{3}\right) \theta\left(x_{3}^{\prime}-a\right) e^{2 i\left(x_{3}^{\prime}-x_{3}\right) \sqrt{\left(p_{\|}\right)^{2}+i \epsilon}} .
$$

We note that the result corresponding to the two half-spaces may also be obtained from the one corresponding to thin mirrors, by performing integrations over two auxiliary variables. Indeed, using the relations:

$$
\theta\left(-x^{3}\right)=\int_{-\infty}^{0} d s_{L} \delta\left(x^{3}-s_{L}\right), \theta\left(x^{3}-a\right)=\int_{a}^{\infty} d s_{R} \delta\left(x^{3}-s_{R}\right)
$$

and that $\Gamma$, for thin mirrors, is only a function of the distance between the mirrors, we obtain:

$$
\Gamma_{I}^{(2)}=\frac{i T \Sigma}{4} \int_{-\infty}^{0} d s_{L} \int_{a-s_{L}}^{\infty} d s_{R} \int d^{3} p_{\|} \frac{e^{2 i s_{R} \sqrt{\left(p_{\|}\right)^{2}+i \epsilon}}}{\left(p_{\|}\right)^{2}+i \epsilon} \tilde{\lambda}_{L}\left(p^{0}\right) \tilde{\lambda}_{R}\left(p^{0}-u p^{1}\right)
$$

or

$$
\begin{aligned}
\Gamma_{I}^{(2)} & =\frac{i T \Sigma}{4} \int_{a}^{\infty} d s_{R} \int_{a-S_{R}}^{0} d s_{L} \int d^{3} p_{\|} \frac{e^{2 i s_{R} \sqrt{\left(p_{\|}\right)^{2}+i \epsilon}}}{\left(p_{\|}\right)^{2}+i \epsilon} \tilde{\lambda}_{L}\left(p^{0}\right) \tilde{\lambda}_{R}\left(p^{0}-u p^{1}\right) \\
& =\frac{i T \Sigma}{4} \int_{a}^{\infty} d s_{R}\left(s_{R}-a\right) \int d^{3} p_{\|} \frac{e^{2 i s_{R} \sqrt{\left(p_{\|}\right)^{2}+i \epsilon}}}{\left(p_{\|}\right)^{2}+i \epsilon} \tilde{\lambda}_{L}\left(p^{0}\right) \tilde{\lambda}_{R}\left(p^{0}-u p^{1}\right) .
\end{aligned}
$$

Since the auxiliary variable is real, one can also extract the imaginary part of $\Gamma$ for the half-spaces from the result corresponding to thin mirrors. Therefore, in what follows we will describe in detail the calculations for the case of thin mirrors, and eventually quote only the final results for half-spaces. To simplify the notation, we will also omit the superscript in the second order approximation to the effective action, that will be denoted by $\Gamma_{I}$.

\section{A. Imaginary part of the in-out effective action}

Since the in-out effective action is related to the vacuum persistence amplitude (Eq. (3)), the presence of an imaginary part signals, for the systems considered in this paper, the excitation of internal degrees of freedom on the mirrors. Since this is due to the constant-velocity motion of one of the mirrors, it reflects the existence of non-contact friction. In this section we will obtain explicit expressions for this imaginary part, for the microscopic model described above, in the case of zero-width mirrors. The case of media filling half-spaces will be considered at the end, taking advantage of the result for thin mirrors.

In what follows, we consider the case of identical mirrors, so we shall drop the $L$ and $R$ subscripts from the microscopic model parameters. If we use the notation $\mathbf{p}_{\|}=\left(p^{1}, p^{2}\right)$, the integrand for the effective action of Eq.(31), 
considered as a function of $p^{0}$, has singularities in $\pm \sqrt{\mathbf{p}_{\|}^{2}-i \epsilon} \approx \pm\left(\left|\mathbf{p}_{\|}\right|-i \epsilon / 2\left|\mathbf{p}_{\|}\right|\right)$. It also has two branch cuts: the first one could be taken as starting on the first singularity, to $+\infty$, parallel to the $x$ axis, (that is, with $\operatorname{Im}\left(p^{0}\right)=$ $-\epsilon / 2\left|\mathbf{p}_{\|}\right|$and $\operatorname{Re}\left(p^{0}\right)>\left|\mathbf{p}_{\|}\right|$, under the approximation of small $\left.\epsilon\right)$. The other branch cut extends parallel to the real axis, from the second singularity to $-\infty$.

In Eq.(31), we can write the integral in the variable $p^{0}$ in the positive axis

$$
\Gamma_{I}=\frac{i T \Sigma}{4} \int d^{2} \mathbf{p}_{\|} \int_{0}^{\infty} d p^{0}\left(f\left(p^{0}\right)+f\left(-p^{0}\right)\right)
$$

where

$$
f\left(p^{0}\right)=\frac{e^{2 i a \sqrt{\left(p^{0}\right)^{2}-\mathbf{p}_{\|}^{2}+i \epsilon}}}{\left(p^{0}\right)^{2}-\mathbf{p}_{\|}^{2}+i \epsilon} \tilde{\lambda}\left(p^{0}\right) \tilde{\lambda}\left(p^{0}-u p^{1}\right)
$$

This allows us to compute the $p^{0}$-integral in the complex plane by considering a closed contour formed by the positive real and imaginary axes and a quarter of a circle with very large radius. As the integral over the quarter of circle vanishes when the radius of the circle tends to infinity, the integral in Eq.(36) is given by its Wick rotated expression $p^{0} \rightarrow i p^{0}$ plus the contribution coming from the poles of $f\left(p^{0}\right)$ in the first quadrant.

As a first example, one may consider the case of constant $\tilde{\lambda}\left(p^{0}\right)$. Denoting this constant by $\omega_{\mathrm{p}}^{2}$, it can be shown that this corresponds to the dielectric permittivity given by the plasma model $\epsilon\left(p^{0}\right)=1-\omega_{\mathrm{p}}^{2} /\left(p^{0}\right)^{2}$. In this case, as $\tilde{\lambda}$ has no poles, and $f\left(i p^{0}\right)$ is a real function, there is no imaginary part in the effective action and therefore no quantum friction.

Let us now consider the case of the microscopic model with uncoupled harmonic oscillators. The function $f\left(p^{0}\right)$ reads

$$
f\left(p^{0}\right)=g^{4} \frac{1}{\left(p^{0}\right)^{2}-\Omega^{2}+i \epsilon} \times \frac{1}{\left(p^{0}-u p^{1}\right)^{2}-\Omega^{2}+i \epsilon} \times \frac{e^{2 i a \sqrt{\left(p^{0}\right)^{2}-\mathbf{p}_{\|}^{2}+i \epsilon}}}{\left(p^{0}\right)^{2}-\mathbf{p}_{\|}^{2}+i \epsilon},
$$

and it has, in addition to the already mentioned singularities, four simple poles, located at:

$$
\begin{aligned}
& p^{0}=\sqrt{\Omega^{2}-i \epsilon} \approx \Omega-\frac{i \epsilon}{2 \Omega} \\
& p^{0}=-\sqrt{\Omega^{2}-i \epsilon} \approx-\Omega+\frac{i \epsilon}{2 \Omega} \\
& p^{0}=u p^{1}+\sqrt{\Omega^{2}-i \epsilon} \approx u p^{1}+\Omega-\frac{i \epsilon}{2 \Omega} \\
& p^{0}=u p^{1}-\sqrt{\Omega^{2}-i \epsilon} \approx u p^{1}-\Omega+\frac{i \epsilon}{2 \Omega} .
\end{aligned}
$$

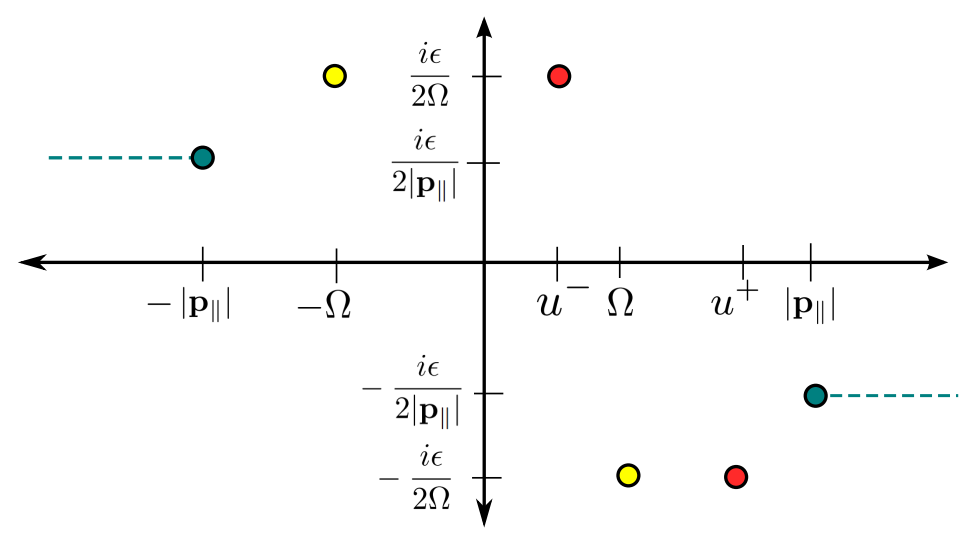

FIG. 1. (Color online). Singularities of $f\left(p^{0}\right)$ (Eq.(38)) in the complex $p^{0}$ plane. Simple poles are depicted as filled dots, while the branch cuts are represented by dashed lines. We have introduced the notation: $u^{ \pm}=u p^{1} \pm \Omega$.

The singularities of $f\left(p_{0}\right)$ are shown in Fig. 1 (the ones for $f\left(-p^{0}\right)$ can be found by $\left.p^{0} \rightarrow-p^{0}\right)$. Note that, for each term of the integrand $\left(f\left(p^{0}\right)\right.$ and $\left.f\left(-p^{0}\right)\right)$, there is only one pole that may appear in the first quadrant, as long as the 
parameters fulfill certain conditions. For the first term, this happens if $u p^{1}-\Omega>0$ while for the second term when $-u p^{1}-\Omega>0$. Then, using Cauchy's theorem we find

$$
\begin{aligned}
\Gamma_{I} & =\frac{i T \Sigma}{4} \int d^{2} \mathbf{p}_{\|} \int_{0}^{+\infty} d p^{0}\left(f\left(p^{0}\right)+f\left(-p^{0}\right)\right)=\frac{-T \Sigma}{4} \int d^{2} \mathbf{p}_{\|}\left\{\int_{0}^{+\infty} d p^{0}\left(f\left(i p^{0}\right)+f\left(-i p^{0}\right)\right)+\right. \\
& \left.+\Theta\left(u p^{1}-\Omega\right) 2 \pi \operatorname{Res}\left[f\left(p^{0}\right), u p^{1}-\sqrt{\Omega^{2}-i \epsilon}\right]+\Theta\left(-u p^{1}-\Omega\right) 2 \pi \operatorname{Res}\left[f\left(-p^{0}\right),-u p^{1}-\sqrt{\Omega^{2}-i \epsilon}\right]\right\} .
\end{aligned}
$$

Noting that $f\left(i p^{0}\right)+f\left(-i p^{0}\right)$ is real, the imaginary part of the effective action becomes:

$\operatorname{Im} \Gamma_{I}=\frac{-T \Sigma \pi}{2} \Im \int d^{2} \mathbf{p}_{\|}\left\{\Theta\left(u p^{1}-\Omega\right) \operatorname{Res}\left[f\left(p^{0}\right), u p^{1}-\sqrt{\Omega^{2}-i \epsilon}\right]+\Theta\left(-u p^{1}-\Omega\right) \operatorname{Res}\left[f\left(-p^{0}\right),-u p^{1}-\sqrt{\Omega^{2}-i \epsilon}\right]\right\}$.

In order to obtain a functional form from the expression above, we evaluate the two residues involved:

$$
\operatorname{Res}\left[\left( \pm p^{0}\right), \pm u p^{1}-\sqrt{\Omega^{2}-i \epsilon}\right]=g^{4} \frac{e^{2 i a} \sqrt{u^{2} p_{1}^{2}+\Omega^{2}-\mathbf{p}_{\|}^{2}-2 u p^{1} \sqrt{\Omega^{2}-i \epsilon}}}{u^{2} p_{1}^{2}+\Omega^{2}-\mathbf{p}_{\|}^{2} \mp 2 u p^{1} \sqrt{\Omega^{2}-i \epsilon}}\left(\frac{1}{u^{2} p_{1}^{2} \mp 2 u p^{1} \sqrt{\Omega^{2}-i \epsilon}}\right)\left(\frac{1}{-2 \sqrt{\Omega^{2}-i \epsilon}}\right)
$$

From Eq. (40), we see that the only modes of the vacuum field that contribute to friction are those with $\left|p_{1}\right|>\Omega / u$. Inserting Eq.(41) into Eq.(40), and performing the change of variables $u p^{1} \rightarrow \omega$, we obtain

$$
\operatorname{Im} \Gamma_{I}=\pi T \Sigma \frac{g^{4}}{2 \Omega} \operatorname{Im}\left\{\int_{-\infty}^{\infty} d p_{2} d \omega \Theta(\omega-\Omega) u \frac{\exp \left[2 i \frac{a}{u} \sqrt{\left(\left(v^{2}-1\right) \omega^{2}+\Omega^{2} u^{2}-p_{2}^{2} u^{2}-2 \omega \Omega u^{2}+i \omega \epsilon u^{2} / \Omega\right.}\right]}{\left.\left(u^{2}-1\right) \omega^{2}+\Omega^{2} u^{2}-p_{2}^{2} u^{2}-2 \omega \Omega u^{2}+i \omega \epsilon u^{2} / \Omega\right) \omega(\omega-2 \Omega+i \epsilon / \Omega)}\right\} .
$$

To perform the integration over $\omega$, we use that

$$
\frac{1}{\omega-2 \Omega+i \epsilon / \Omega}=\operatorname{p.v} \cdot\left(\frac{1}{\omega-2 \Omega}\right)-i \pi \delta(\omega-2 \Omega)
$$

and note that $\epsilon$ can be set to zero in the rest of the integrand. Performing another change of variables, $u p_{2} a \rightarrow x$, the final expression for the effective action reads

$$
\operatorname{Im} \Gamma_{I}=\frac{\pi^{2}}{4} \frac{T \Sigma}{a^{3}} \frac{g^{4}}{\Omega^{6}}(\Omega a)^{4} \int_{-\infty}^{\infty} d x \frac{e^{-\frac{2}{u} \sqrt{(\Omega a)^{2}\left(4-u^{2}\right)+x^{2}}}}{(\Omega a)^{2}\left(4-u^{2}\right)+x^{2}} \simeq \frac{\pi^{2}}{4} \frac{T \Sigma}{a^{3}} \frac{g^{4}}{\Omega^{6}}(\Omega a)^{4} \int_{-\infty}^{\infty} d x \frac{e^{-\frac{2}{u} \sqrt{4(\Omega a)^{2}+x^{2}}}}{4(\Omega a)^{2}+x^{2}}
$$

This is the main result of this section, written as a product of dimensionless factors. The integral over $x$ on the Eq.(43) may be performed numerically. In Fig. 2 we show the result for the imaginary part of the effective action as a function of $u$, for $\Omega a=0.01$. As expected, the dissipative effects are strongly suppressed as $u \rightarrow 0$. Indeed, the integral in Eq. (43) vanishes as $\exp (-4 \Omega a / u)$ for $u \ll \Omega a$, and grows linearly in $u$ in the opposite limit.

The imaginary part of the effective action for half-spaces can be obtained by integrating the thin mirrors case, as explained above (see Eq.(35)). The result is

$$
\operatorname{Im} \Gamma_{I}=\frac{\pi^{2}}{16} \frac{T \Sigma}{a^{3}} \frac{g^{4}}{\Omega^{8}}(\Omega a)^{6} u^{2} \int_{-\infty}^{\infty} d x \frac{e^{-\frac{2}{u} \sqrt{(\Omega a)^{2}\left(4-u^{2}\right)+x^{2}}}}{\left[(\Omega a)^{2}\left(4-u^{2}\right)+x^{2}\right]^{2}} .
$$

As already mentioned, the coupling constants $g$ for half-spaces and thin mirrors have different dimensions. We show the numerical results for this integration in Fig. 3, as a function of $u$, with $\Omega a=0.01$. As in the previous case, there is a strong suppression for low velocities.

\section{FRICTIONAL FORCE}

Since $\operatorname{Im} \Gamma_{I}>0$ when the mirrors are in relative motion, there is an energy transfer to the system. Indeed, starting in the in vacuum $\left|0_{i n}\right\rangle$, the system ends up being in an excited state, as can be seen from the vacuum persistence probability

$$
\left|\left\langle 0_{\text {out }} \mid 0_{\text {in }}\right\rangle\right|^{2}=e^{-2 \operatorname{Im} \Gamma_{I}}
$$




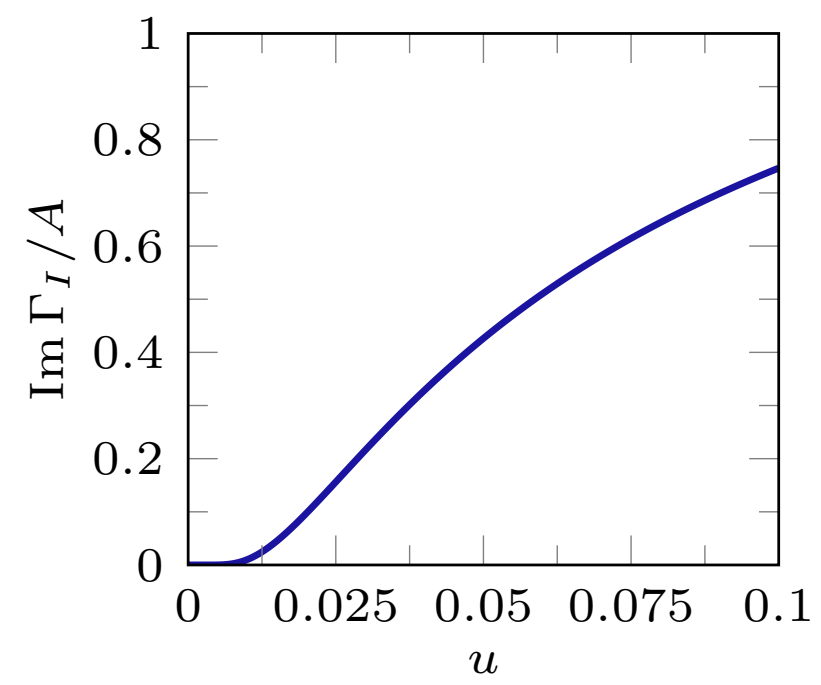

FIG. 2. (Color online). Imaginary part of the effective action for thin mirrors, as a function of $u$, with $\Omega a=0.01$. $A$ is the global factor $A=\frac{g^{4} T \Sigma(\Omega a)^{3} \pi^{2}}{4 a^{3} \Omega^{6}}$. The imaginary part of the effective action, and hence the dissipative effects, are strongly suppressed for small values of the velocity between the plates.

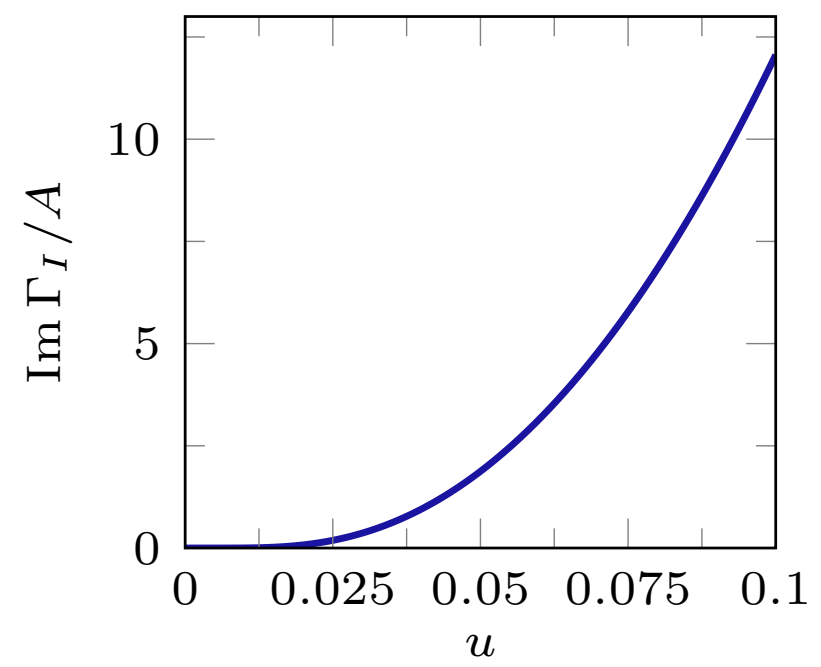

FIG. 3. (Color online). Imaginary part of the effective action for half-spaces, as a function of $u$, with $\Omega a=0.01$. $A$ is the global factor $A=\frac{g^{4} T \Sigma(\Omega a)^{5} \pi^{2}}{16 a^{3} \Omega^{8}}$. The imaginary part of the effective action, and hence the dissipative effects, are strongly suppressed for small values of the velocity between the plates.

Therefore, energy conservation implies that there should be force performing mechanical work when moving the mirror. Moreover, since this motion has a constant speed, the force has to be dissipative in nature. In spite of the fact that the effective action allows one to understand, in an indirect way, the existence of a frictional force, it is not the appropriate tool to find that force explicitly.

On the other hand, we believe that it is important, as a consistency check, to have an explicit, independent evaluation of that frictional force. To find that expression, we compute the mean value of the energy-momentum tensor $t_{\mu \nu}$ in the in-vacuum, in the steady regime:

$$
\left\langle t_{\mu \nu}\right\rangle \equiv\left\langle 0_{\text {in }}\left|t_{\mu \nu}\right| 0_{\text {in }}\right\rangle
$$

The force per unit area, $\sigma$ can be obtained by means of the point-splitting technique:

$$
\sigma=\lim _{x \rightarrow a^{+}}\left\langle t_{13}(x)\right\rangle-\lim _{x \rightarrow a^{-}}\left\langle t_{13}(x)\right\rangle,
$$


where

$$
\left\langle t_{13}(x)\right\rangle=\lim _{x^{\prime} \rightarrow x}\left\langle\partial_{1} \phi(x) \partial_{3}^{\prime} \phi\left(x^{\prime}\right)\right\rangle=\frac{1}{2} \lim _{x^{\prime} \rightarrow x} \int \frac{d p^{0}}{2 \pi} \frac{d^{2} p_{\|}}{(2 \pi)^{2}}\left(i p_{1}\right) \partial_{3}^{\prime} G_{1}\left(p^{0}, p_{\|}, x_{3}, x_{3}^{\prime}\right) .
$$

Here, $G_{1}$ denotes Hadamard's two-point function, that is defined by:

$$
G_{1}\left(x, x^{\prime}\right)=\left\langle 0_{\text {in }}\left|\left\{\phi(x), \phi\left(x^{\prime}\right)\right\}\right| 0_{\text {in }}\right\rangle .
$$

Note that the very fact that there is a non-vanishing imaginary part in $\Gamma$ implies that the in-vacuum is different from the out-vacuum; thus, in order to compute the mean value of the energy momentum tensor one cannot use the in-out formalism. It is well-known, however, that one can use the the Schwinger-Keldysh, CTP, or in-in approach [10]. We note that this point becomes irrelevant when computing the static Casimir force, since when $u=0$ the two vacua are equivalent.

In the usual in-out formalism, the Feynman propagator in the presence of the mirrors can be computed perturbatively, assuming that the potentials $V_{R}$ and $V_{L}$ are small perturbations to the free problem. We have, schematically,

$$
G_{F}=G_{F}^{(0)}+G_{F}^{(0)} V_{L} G_{F}^{(0)} V_{R} G_{F}^{(0)}+L \leftrightarrow R,
$$

where we only included terms with mixed contributions from the $\mathrm{L}$ and $\mathrm{R}$ mirrors. We also omitted the integrations in the contraction of the propagators.

In the CTP formalism, the free propagator is a $2 \times 2$ matrix with elements $G_{\alpha \beta}^{(0)}$, where $\alpha, \beta=+,-:$ [10]

$$
G_{\alpha \beta}^{(0)}(p) \equiv\left(\begin{array}{cc}
1 /\left(p^{2}+i \epsilon\right) & 2 \pi \delta\left(p^{2}\right) \theta\left(-p^{0}\right) \\
2 \pi \delta\left(p^{2}\right) \theta\left(p^{0}\right) & 1 /\left(p^{2}-i \epsilon\right)
\end{array}\right)
$$

It is worth noting that $G_{++}^{(0)}$ is defined by:

$$
G_{++}\left(x, x^{\prime}\right)=\left\langle 0_{\text {in }}\left|T \phi(x) \phi\left(x^{\prime}\right)\right| 0_{\text {in }}\right\rangle,
$$

and it is related to the Hadamard's function by $G_{1}\left(x, x^{\prime}\right)=-2 \operatorname{Im}\left(G_{++}\left(x, x^{\prime}\right)\right)$.

The CTP version of the perturbative evaluation of the propagator is

$$
G_{++}=G_{++}^{(0)}+G_{+\alpha}^{(0)} V_{L, \alpha \beta} G_{\beta \gamma}^{(0)} V_{R, \gamma, \delta} G_{\delta,+}^{(0)}+L \leftrightarrow R,
$$

where the potentials $V_{L, R}$ are again $2 \times 2$ matrices

$$
\lambda\left(p^{0}\right)=\left(\begin{array}{cc}
1 /\left(\left(p^{0}\right)^{2}-\Omega^{2}+i \epsilon\right) & -\frac{\pi}{\Omega} \delta\left(p^{0}+\Omega\right) \\
-\frac{\pi}{\Omega} \delta\left(p^{0}-\Omega\right) & 1 /\left(\left(p^{0}\right)^{2}-\Omega^{2}-i \epsilon\right)
\end{array}\right) .
$$

Computing explicitly every contraction in Eq.(53), the desired component of the energy-momentum tensor may be written as:

$$
\left\langle t_{13}(x)\right\rangle=-\operatorname{Im}\left\{\lim _{x \rightarrow x^{\prime}}\left[\partial_{1} \partial_{3}^{\prime} G_{++}^{(0)}\left(x, x^{\prime}\right)+\int d u d v d y d z \partial_{1} G_{+\alpha}^{(0)}(x, u) V_{L, \alpha \beta}(u, v) G_{\beta \gamma}^{(0)} V_{R, \gamma \delta}(y, z) \partial_{3}^{\prime} G_{\delta+}^{(0)}\left(z, x^{\prime}\right)\right]\right\},
$$

where we have written explicitly the spatial integrations. The dissipative force is given by the discontinuity of the previous magnitude at $x=a$. The first term in Eq.(55), the contribution of the free vacuum field propagator, is continuous at $x=a$ and will not contribute to the force. Writing the free propagators in momentum space, the derivatives can easily be calculated, and it can be shown that the only non-vanishing contribution to the force comes from the term with $\delta=+$, for the $\delta=-$ is continuous at $x=a$. The force is then given by:

$$
\sigma=\operatorname{Im} \int \frac{d p^{0}}{2 \pi} \frac{d^{2} p_{\|}}{(2 \pi)^{2}} i p_{1} G_{+\alpha}^{(0)}\left(p^{0}, p_{\|}, a\right) V_{L, \alpha \beta}(p) G_{\beta \gamma}^{(0)}\left(p^{0}, p_{\|}, a\right) V_{R, \gamma+}(p)+L \leftrightarrow R .
$$

The integrand consists of eight different terms, but only one of them turns out to be non-vanishing: the one with $\alpha=+, \beta=\gamma=-$. The other seven terms vanish either due to parity considerations, or as a result of the Heaviside and Dirac delta functions appearing on the propagators and potentials. The remaining term can easily be calculated since the integration over $p^{0}$ and $p_{1}$ is trivial thanks to the Dirac and Heaviside functions. The final result is

$$
\sigma=-\frac{1}{4 \pi a^{4}} \frac{g^{4}}{\Omega^{6}}(\Omega a)^{6} \frac{1}{u} \int_{-\infty}^{\infty} d x \frac{e^{-\frac{2}{u} \sqrt{(\Omega a)^{2}\left(4-u^{2}\right)+x^{2}}}}{(\Omega a)^{2}\left(4-u^{2}\right)+x^{2}}
$$


This is the main result of this section (we have written the result as a factor $1 / a^{4}$, which gives the units of $\sigma$, times dimensionless factors). Note that we are left with the exact same integral that we found while calculating the effective action, which is not surprising, since both quantities account for the dissipative effects present on the system. This expression can be numerically integrated, and the result is shown in Fig. 4 . The behaviour of the force as a function of the relative velocity of the plates shows that, as expected, quantum friction is practically negligible for very small velocities $u \ll \Omega a$.

It is worth to remark that, had we naively used the in-out or Euclidean formalisms, we would have obtained a vanishing value for the frictional force.

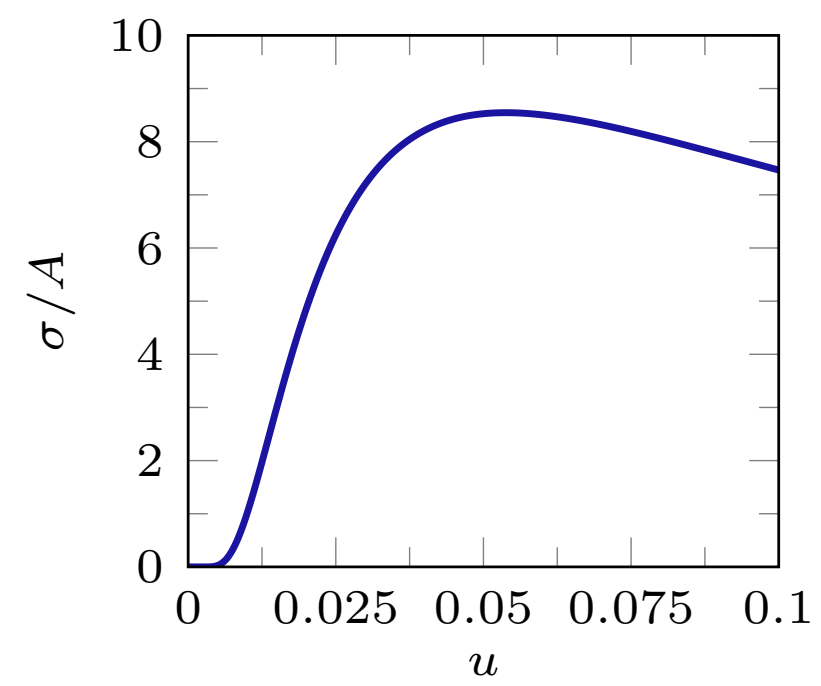

FIG. 4. (Color online). Modulus of the dissipative force, as a function of the relative velocity of the plates, for $\Omega a=0.01$. The global factor is $A=\frac{g^{4}(\Omega a)^{5}}{4 \Omega^{6} \pi a^{4}}$. The frictional force is practically negligible for small values of the velocity between the plates.

It is now a matter of performing an integration to find the force corresponding to half-spaces, since the same argument used for the effective action applies here. Thus, for two half-spaces, we have:

$$
\begin{aligned}
\sigma & =-\frac{g^{4}}{4 \pi \Omega u} \int_{a}^{\infty} d s(s-a) \int d k \frac{\exp \left[-\frac{2 s}{u} \sqrt{\Omega^{2}\left(4-u^{2}\right)+k^{2}}\right]}{\Omega^{2}\left(4-u^{2}\right)+k^{2}} \\
& =-\frac{1}{16 \pi a^{4}} \frac{g^{4}}{\Omega^{8}}(\Omega a)^{7} u \int d x \frac{\exp \left[-\frac{2}{u} \sqrt{(\Omega a)^{2}\left(4-u^{2}\right)+x^{2}}\right]}{\left[(\Omega a)^{2}\left(4-u^{2}\right)+x^{2}\right]^{2}},
\end{aligned}
$$

a result which is shown in Figure 5. We recall that the mass dimensions of $g$ and $\Omega$ are different when the mirrors have a non-zero width, as explained in II B.

\section{CONCLUSIONS}

In this paper we have used a functional approach to study quantum friction effects on imperfect moving mirrors, within a model where a scalar vacuum field is coupled to microscopic degrees of freedom confined to two mirrors, moving with a constant relative speed. This coupling induces, after integrating out the microscopic degrees of freedom, a nonlocal interaction term in the action for the vacuum field, having an structure which depends on the relative velocity between the mirrors. The nonlocal action for the vacuum field has been used to approach the problem from two complementary viewpoints.

In the first part of the paper, we computed the imaginary part of the in-out effective action. Being related to the vacuum persistence amplitude, the presence of an imaginary part is a signal for dissipative effects. In order to clarify the relation between dissipation and the analytic properties of the nonlocal interaction (i.e. of the analogue of the dielectric permittivity), we performed a detailed analysis in real time. The integration of the microscopic degrees

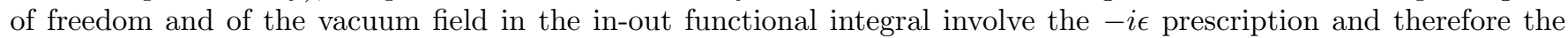
presence of the Feynman propagator, whose analytic structure determines both the analytic structure of the dielectric permittivity and of the full effective action, implying the existence of quantum friction effects. The analysis of the 


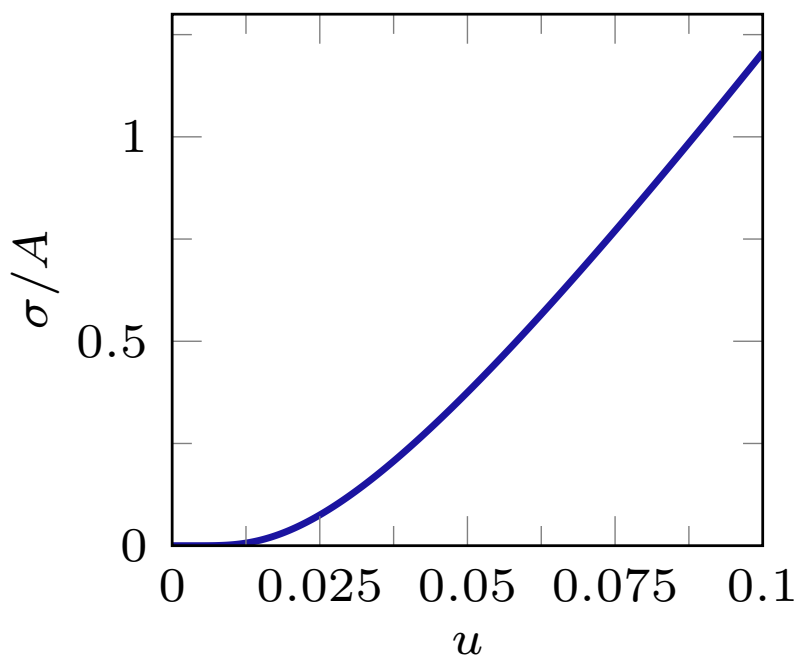

FIG. 5. (Color online). Modulus of the dissipative force for half-spaces, as a function of the relative velocity of the plates, for $\Omega a=0.01$. The global factor is $A=\frac{g^{4}(\Omega a)^{5}}{16 \Omega^{8} \pi a^{4}}$. The frictional force is practically negligible for small values of the velocity between the plates.

present paper complements the Euclidean approach of Ref.[8] and clarifies the issue of the validity of the Wick rotation of the Euclidean results.

In the second part of the paper, we computed the frictional force between mirrors. In order to do this, we used the CTP formalism, which is crucial to obtain the correct result for $\left\langle 0_{\text {in }}\left|t_{\mu \nu}\right| 0_{\text {in }}\right\rangle$. The crucial point here is that, due to dissipation, the in and out vacuum states are different. This is the reason why the CTP formalism is not required to compute static Casimir forces, while its use is unavoidable to compute the force on moving mirrors [9].

The approach described here can be generalized to the more realistic case of the electromagnetic field at non vanishing temperature. The nonlocal interaction should be generalized accordingly, and will involve the derivatives of the potential vector $A_{\mu}$ on the position of the mirrors. Work in this direction is in progress.

\section{ACKNOWLEDGEMENTS}

This work was supported by ANPCyT, CONICET, UBA and UNCuyo.

[1] P.W. Milonni, The Quantum Vacuum (Academic Press, San Diego, 1994); M. Bordag, U. Mohideen, and V.M. Mostepanenko, Phys. Rep. 353, 1 (2001); K. A. Milton, The Casimir Effect: Physical Manifestations of the Zero- Point Energy (World Scientific, Singapore, 2001); S. Reynaud, A. Lambrecht, C. Genet, and M.T. Jaekel, et al., C. R. Acad. Sci. Paris Ser. IV 2, 1287 (2001); K. A. Milton, J. Phys. A 37, R209 (2004); S. K. Lamoreaux, Rep. Prog. Phys. 68, 201 (2005); M. Bordag, G. L. Klimchitskaya, U. Mohideen, and V. M. Mostepanenko, Advances in the Casimir Effect (Oxford University Press, Oxford, 2009).

[2] J.B. Pendry, J. Phys.:Condens. Matter 9, 10301 (1997).

[3] M. F. Maghrebi, R. Golestanian and M. Kardar, Phys. Rev. A 88, 042509 (2013).

[4] J.B. Pendry, New J. Phys. 12, 033028 (2010); ibidem New J. Phys. 12, 068002 (2010); T.G. Philbin and U. Leonhardt, New J. Phys. 11, 033035 (2009); U. Leonhardt, New J. Phys. 12, 068001 (2010).

[5] J.S. Hoye and Brevik I., EPL, 91, 60003 (2010); ibidem Europ. Phys. J. D, in press (arXiv:1009.3135 v2); G. Barton, New J. Phys. 12, 113044 (2010); ibidem New J. Phys. 12, 113045 (2010).

[6] A.I. Volokitin and B.N.J. Persson, Rev. Mod. Phys. 79, 1291 (2007).

[7] For recent reviews see V. V. Dodonov, Phys. Scripta 82 (2010) 038105; D. A. R. Dalvit, P. A. Maia Neto and F. D. Mazzitelli, Lect. Notes Phys. 834 (2011) 419; P. D. Nation, J. R. Johansson, M. P. Blencowe and F. Nori, Rev. Mod. Phys. 84, 1 (2012).

[8] C. D. Fosco, F. C. Lombardo and F. D. Mazzitelli, Phys. Rev. D 84, 025011 (2011) [arXiv:1105.2745v1 [hep-th]].

[9] C. D. Fosco, F. C. Lombardo and F. D. Mazzitelli, Phys. Rev. D 76, 085007 (2007) [arXiv:0705.2960 [hep-th]]. 
[10] J. S. Schwinger, J. Math. Phys. (N.Y.) 2, 407 (1961); L. V. Keldysh, Zh. Eksp. Teor. Fiz. 47, 1515 (1965) [Sov. Phys. JETP 20, 1018 (1965)] E. A. Calzetta and B. L. Hu, Nonequilibriium Quantum Field Theory (Cambridge University Press, Cambridge, England, 2008). 